\title{
Comparison of imaging follow-up between joints with arthroscopic surgery (lysis and lavage) and those with non-surgical treatment
}

Hiroshi Kurita, DDS, PhD, ${ }^{*}$ Zhao Chen, MD ${ }^{\dagger}$ Shinobu Uehara, DDS, Hideki Miyazawa, DDS, ^ Kenji Kurashina, DDS, $\mathrm{PhD}^{\jmath}$

* Associate professor, Department of Dentistry and Oral Surgery, Shinshu University School of Medicine

$\uparrow$ Postgraduate student, Department of Dentistry and Oral Surgery, Shinshu University School of Medicine

I Lecturer, Department of Dentistry and Oral Surgery, Shinshu University School of Medicine

$\int$ Professor in chief, Department of Dentistry and Oral Surgery, Shinshu University School of Medicine

Corresponding author: Hiroshi Kurita

Department of Dentistry and Oral Surgery

Shinshu University School of Medicine

3-1-1, Asahi, Matsumoto, 390-8621, Japan

Tel. +81(0)263 37 2677, Fax. ;81(0)263 372676

e-mail; hkurita@hsp.md.shinshu-u.ac.jp 


\begin{abstract}
:
Purpose: To assess the influence of arthroscopic surgery on radiographically evident degenerative change of the temporomandibular joint (TMJ). The posttreatment course was compared between the joints that underwent arthroscopic lysis and lavage and those that underwent nonsurgical treatment.
\end{abstract}

Patients and Methods: Twenty-eight patients agreed to imaging follow-up examination of 35 joints and were included in this study. Twenty-four joints of 19 patients underwent only nonsurgical treatment (nonsurgical joints). While, eleven joints of nine patients had failed the nonsurgical treatment and consequently underwent arthroscopic lysis and lavage (arthroscopic joints). The joints were assessed at first visit and at least 20 months later (mean 79 months) for disk displacement, disk position, disk morphology, disk mobility, condylar morphology, morphology of the articular eminence, and horizontal condylar angle and size. Thereafter, interval change was assessed and compared between the groups.

Results: There was no significant difference in the prevalence of the progressive degenerative changes between the groups. In addition, there was no significant difference in the change of size and morphology of the condyle. However, a higher prevalence of improvement of disk mobility in the arthroscopic joints rather than the nonsurgical joints was significant (Goodness test of fit for chi-square, $\mathrm{p}<0.05$ ).

Conclusions: The results of this study suggest that the posttreatment course of radiographically evident degenerative change was not significantly different between arthroscopy and nonsurgical treatment; however, arthroscopic surgery showed a greater ability to improve disk mobility. In imaging follow-up, arthroscopic lysis and lavage is a minimally invasive treatment modality that is equivalent to nonsurgical treatment.

Key words: TMJ, arthroscopic surgery, nonsurgical treatment, MRI, degenerative change, follow-up 


\section{Introduction}

In the treatment of temporomandibular joint (TMJ) diseases, arthroscopic surgery has been shown to be an effective and safe treatment modality. Significant improvement in the symptoms and in the range of mandibular movement in patients with severe and recalcitrant symptoms related to TMJ disorders with some degree of degenerative changes have been reported. ${ }^{1-3}$ Arthroscopic surgery of the TMJ has been introduced as a minimally invasive procedure. However, little is known about the long-term course of radiographically evident degenerative changes of TMJ after arthroscopic surgery. ${ }^{4}$ Invasive treatment modalities might directly affect the degenerative change of the articular components of TMJ.

The purpose of this study was to assess the influence of arthroscopic surgery on degenerative change of the articular components of the temporomandibular joint (TMJ). TMJs were examined using magnetic resonance imaging (MRI) before and after treatments for TMJ disorders. The results of imaging follow-up were compared between the joints that had undergone arthroscopic surgery and those treated with nonsurgical treatments.

\section{Materials and Methods}

The subjects were based on a consecutive series of 181 patients who underwent magnetic resonance (MR) imaging of TMJs during the period between July 1991 and December 2000. Of these, 28 patients accepted an invitation for imaging reassessments of their TMJs. Seven patients had bilateral and 21 patients had unilateral joint disease and, therefore, 35 joints of 28 patients were available for the study. Twenty-three were women and five were men. The mean age at their fist visit was 36.3 (SD 15.1) years. The subjects were divided into two groups according to the treatments modalities employed. Twenty-four joints of 19 patients had undergone one or two nonsurgical treatment modalities including a stabilization-type occlusal appliance, a disk repositioning-type occlusal appliance, medication, and/or a disk recapturing-type occlusal appliance and were classified into the conservative-treatment group (nonsurgical joints). Eleven joints of nine patients failed the conservative treatments 
for more than three months and consequently underwent arthroscopic lysis and lavage, and they were assigned to the arthroscopic-surgery group (arthroscopic joints). All treatments were completed within a year after the patients' first visit.

The imaging examinations were carried out within a few weeks after the patient's first visit and at the time of recall. The subjects were assessed for disk displacement, disk position, disk morphology, disk mobility, OA change of the condyle, resorption of the lateral part (postero-superior aspect of the lateral pole) of the condyle, morphology of the articular eminence, horizontal condylar angle, and horizontal condylar size using MR imaging and a plane radiograph. MRI was performed with 1.5-tesla system (Sigma Advantage, General Electric Medical Systems, Milwaukee, WI, USA) using a TMJ surface coil. An initial axial localizer (TR $300 \mathrm{~ms}$, TE $16 \mathrm{~ms}$, FOV $24 \mathrm{~cm}, 5-\mathrm{mm}$ slice thickness, $256 \times 192$ scanning matrix) that was nearly parallel to the Frankfort horizontal plane and could maximally visualize the lateral and medial poles of both condyle was obtained in the closed-mouth position. Five 3-mm slice thickness, oblique sagittal images of the TMJ (TR $500 \mathrm{~ms}$, TE $15 \mathrm{~ms}$, FOV $16 \mathrm{~cm}$, a 256 x 192 scanning matrix) were obtained with the jaws in the maximal intercuspal position and then at maximal opening.

Oblique sagittal MR images were assessed for disk displacement, disk position, disk morphology, disk mobility, OA change of the condyle, and morphology of the articular eminence at a representative center depth of the TMJ. Disk displacement was defined as previously reported in the central section of sagittal MR images of the $\mathrm{TMJ}^{5,6}$ and was classified as normal (no displacement), mild (disk displacement with reduction), and severe (disk displacement without reduction). Disk position was measured on sagittal MR image representing the center depth of the TMJ using the method previously reported (Fig 1A). ${ }^{7}$ Disk morphology was clarified in the closed-mouth projection in the sagittal plane and classified as normal (biconcave-shape), mild (biplanar or convex-shape), or severe (folded-shape or amorphous). ${ }^{8}, 9$ The mobility of the disk was determined 
in the closed-and opened-mouth projection in the sagittal plane. The disk mobility was classified as mobile (normal) if the disk changed its position relative to the glenoid fossa and the articular eminence. Disk fixation was considered to be present in case of lack of the position change. ${ }^{10}$ OA change of the condyle was considered to be present if erosive and/or proliferative changes, including flattening, spurring or eburnation, were found in the sagittal MR images of TMJ. ${ }^{11}$ The morphology of the articular eminence was classified into two types, the box/sigmoid type and the flattened/deformed type, according to the criteria reported previously (Fig 1B). ${ }^{5}$ OA change of the articular eminence was considered to be present if flattened or deformed type was found.

Resorption of the lateral part (postero-superior aspect of the lateral pole) of the mandibular condyle was assessed as described by Kurita et al. ${ }^{12}$ That is, a plane radiograph of the transorbital oblique frontal projection was taken during maximum mouth opening, and the resorption of the lateral part of the condyle was considered to be present if OA changes including bony surface erosion, concavity or flattening were observed at the postero-superior corner of the lateral part of the condyle.

The horizontal angle of the condyle was measured on axial MR images (localizer) using the methods reported by Westesson et al (Fig 1C). 13, 14 The horizontal condylar size was measured in the antero-posterior and medio-lateral dimensions on axial MR images (localizer) using the previously reported methods (Fig 1D). ${ }^{15}$ Each image was separately evaluated by a trained radiologist and one of the authors (HK). Any disagreements were discussed until consensus was reached. The measurement was carried out twice by one of the authors (HK) blinded to the clinical and other result of TMJ imaging. The final value was obtained by calculating the mean value of the two measurements.

In each joint, the result of imaging assessment was compared between the initial and the follow-up examination, and the interval change was judged and classified into three categories: "improvement", "no change", and "progress". The joint was considered improved if the 
change disappeared or improved from severe to mild. The joint was considered as having no change if there was no interval change. "Progress" was reported if the change newly appeared or progressed from mild to severe. Thereafter, the prevalence of interval change was compared between the joints with arthroscopic surgery and those with nonsurgical treatment, and the goodness test of fit for the chi-square was used to test a statistical difference. The differences in the disk position, the horizontal condylar angle, and the horizontal condylar size between the initial examination and the follow-up imaging was calculated as "the value in the initial imaging" minus "the value in the follow-up imaging", and was compared between the two groups. Mann-Whitney's test was used to determine statistical difference. The statistical analysis was carried out using computer software (StatView 5.0, SAS Institute Inc., NC). P $<0.05$ was considered significant.

\section{Results}

The average interval between the first visit and the time of imaging reassessment was 79 months with a range of 20 to 137 months. Comparison of characteristics between the arthroscopic and the nonsurgical joints is summarized in Table 1. The time interval of the nonsurgical joints was significantly longer than that of the arthroscopic joints (Student's t-test, $\mathrm{p}<0.05$ ). There was a significantly higher prevalence of the fixed TMJ disk in the arthroscopic joints than in the nonsurgical joints at the initial MRI examination (Fisher's exact probability test, $\mathrm{p}<0.05)$. In addition, according to the criteria reported by American Association of Oral and Maxillofacial Surgeons (1992) ${ }^{16}$, all patients who underwent only nonsurgical treatments were satisfied with treatment outcome, while three out of nine patients complained of some slight remaining symptoms. The difference in the treatment outcome was statistically significant (Fisher's protected least significant difference, $\mathrm{p}<0.05)$. 
Comparisons of the interval change in disk displacement, disk morphology, disk mobility, OA change of the condyle, OA change of the articular eminence, and resorption of the lateral part of the condyle between the two groups are summarized in Table 2. A statistically significant difference was observed only in disk mobility. A higher prevalence of improvement of disk mobility in the arthroscopic rather than the nonsurgical joints was significant (Goodness test of fit for chi-square, $\mathrm{p}<0.05)$. There was no other significant difference between them.

Comparisons of the calculated amount of changes in disk position, horizontal condylar angle, and horizontal size are shown in Table 3. In each variable, the interval change was negligible and there was no significant difference between the arthroscopic and the nonsurgical joints (Mann-Whitney`s U-test, $\mathrm{p}>0.05$ ).

\section{Discussion}

The purpose of this study was to assess the posttreatment course of radiographically evident degenerative change in joints that underwent arthroscopic lysis and lavage. The results of imaging follow-up were compared between the joints that underwent arthroscopic lysis and lavage and those that underwent nonsurgical treatments only.

In the results of this study, the time interval between the first and the follow-up examination was significantly longer in the joints that had undergone arthroscopic surgery than in those with nonsurgical treatments. Therefore, a long time interval might influence the incidence and severity of the degenerative changes of TMJ. In this study, when the relationship between the length of follow-up and either the calculated amount of change in the disk position, the horizontal condylar angle, or the horizontal condylar size was analyzed, no significant correlation was found (Pearson's correlation coefficient, $p>0.05$ ). Our previous study also showed that the difference in the follow-up period had no significant influence on the incidence and severity of the degenerative change of the 
TMJ. ${ }^{17}$ Some authors described the length of ongoing radiographic changes to last a few years, after which period a stable terminal stage was reached. ${ }^{17-19}$ In this study, the median follow-up period for the arthroscopic joints was 30 months with the inter quartile range of 32 months. In addition, all joints, except one, were reevaluated at least two years after the initial examination. Therefore, we hypothesize that the difference in the follow-up period of more than a few years in the studied subjects did not have a significant influence on the results of this study.

Previously, Ohnuki et $\mathrm{l}^{4}{ }^{4}$ studied MR imaging of TMJ disks before and after arthroscopic surgery for TMJ disorders, and reported that deformity of the disks progressed and disk mobility increased after arthroscopic surgery. In this study, the most frequently observed progressive change after arthroscopic surgery was the morphological change of the TMJ disk. The disk morphologically progressed in 5 out of 11 joints in the arthroscopic joints and our result was consistent with their result. However, our previous study assessed the long-term course of radiographically evident degenerative changes of TMJ after nonsurgical treatments and showed that disk deformity progressed significantly during the intervening years. ${ }^{17,20}$ The present study revealed that disk morphology progressed in 9 out of 24 joints in the nonsurgical joints and there was no significant difference in progression or improve rates between the joints treated with arthroscopic surgery or nonsurgical treatments. These results suggest that progress in the disk morphology was not a characteristic change for the joints after the arthroscopic surgery.

Concerning disk mobility, a higher prevalence of disk fixation was observed in the joints that required arthroscopic surgery than in the joints where a successful outcome was obtained with nonsurgical treatments. This indicates that the joints with a fixed disk seem to be refractory to nonsurgical treatments. The results of this study showed that disk mobility increased more frequently after arthroscopic surgery than after nonsurgical treatments. This result was consistent with the result of 
Ohnuki et $\mathrm{al}^{4}$. They also reported that improvement of disk mobility was observed after arthroscopic surgery, especially in the joints with successful outcome. In the success group, they reported, every joint had a mobile disk after arthroscopic surgery. In the results of this study, two out of three joints in which a fair outcome was obtained after the arthroscopic surgery showed persistent immobility of TMJ disk through the arthroscopic surgery. As stated by some researchers, TMJ arthroscopy gives good results in treating patients with anchored disc phenomenon. ${ }^{21}$, 22 These results suggest that improvement of disk mobility was a positive change that was produced by the arthroscopic surgery (lysis and lavage).

The results of this follow-up study showed that there was no significant difference in the prevalence of progressive degenerative change between the joints treated with arthroscopic surgery and those treated with nonsurgical treatments. Furthermore, there was no significant difference in the change of the size and morphology of the condyle. In both groups, the interval changes of the soft and hard TMJ tissues were slight and mild, while disk deformation significantly progressed. Murakami et al ${ }^{23}$ evaluated the long-term outcome after arthroscopic surgery for patients with a severe stage of internal derangement and reported that radiographic examination revealed mild or moderate degenerative changes but no severe progressive changes. These results suggest that radiographic alteration after arthroscopic surgery was mild and it was not significantly different from that after nonsurgical treatment.

In conclusion, the posttreatment course in radiographically evident degenerative change of the TMJ soft and hard tissues was slight and mild in the joints with arthroscopic surgery as well as in those with nonsurgical treatments. Degenerative change after arthroscopic surgery showed the same incidence and severity as that after nonsurgical treatments, while arthroscopic surgery revealed a greater ability to improve the mobility of the TMJ disk. We postulate that arthroscopic 
lysis and lavage may not have a direct effect on the degenerative change of the articular components of TMJ. 


\section{REFERENCES}

1. McCain JP, Sanders B, Koslin MG, Quinn JH, Peters PB, Indresano AT, Quinn JD. Temporomandibular joint arthroscopy: a 6-year multicenter retrospective study of 4,831 joints. J Oral Maxillofac Surg 50:926, 1992 .

2. Murakami K, Segami N, Okamoto M, Yamamura I, Takahashi K, Tsuboi Y. Outcome of arthroscopic sugery for internal derangement of the temporomandibular joint: long-term results covering 10 years. J Cranio-Maxillofac Surg 28:264, 2000.

3. Perrott DH, Alborzi A, Kaban LB, Helms CA. A prospective evaluation of the effectiveness of temporomandibular joint arthroscopy. J Oral Maxillofac Surg 48:1029, 1990.

4. Ohnuki T, Fukuda M, Iino M, Takahashi T. Magnetic resonance evaluation of the disk before and after arthroscopic surgery for temporomandibular joint disorders. Oral Surg Oral Med Oral Pathol Oral Radiol Endod 96:141, 2003.

5. Kurita H, Ohtsuka A, Kobayashi H, Kurashina K. Is the morphology of the temporal component of the temporomandibular joint a predisposing factor for disk displacement? Dentomaxillofac Radiol 29: 159, 2000:.

6. Rao VM, Liem MD, Faroke A, Razek AAKA. Elusive "stuck" disk in the temporomandibular joint: diagnosis with MR imaging. Radiology 189: $823,1993$.

7. Kurita H, Kurashina K, Ohtsuka A, Kotani A. A change of position of the temporomandibular joint disk with insertion of a disk repositioning appliance. Oral Surg Oral Med Oral Pathol Oral Radiol Endod 85:142, 1998.

8. Katzberg RW. Temporomandibular joint imaging. Radiology 170: $297,1989$.

9. Hasson AN, Alder ME, Knepel KA. Magnetic resonance imaging. In: Cristiansen EL, Thompson JR, editors. Temporomandibular joint imaging. St.Louis, Mosby, 1990, p 147. 
10. Rao VM, Liem MD, Faroke A, Razek AAKA. Elusive "stuck" disk in the temporomandibular joint: diagnosis with MR imaging. Radiology 189: 823, 1993.

11. Rao VM, Babaria A, Manoharan A, Mandel S, Gottehrer N, Wank H, Grosse S. Altered condylar morphology associated with disk displacement in TMJ dysfunction: observations by MRI. Magn Reson Imaging 8:231, 1990 .

12. Kurita H, Ohtsuka A, Kobayashi H, Kurashina K. Resorption of the lateral pole of the mandibular condyle in temporomandibular disc displacement. Dentomaxillofac Radiol 30: 88, 2001.

13. Westesson P-L, Bifano JA, Tallents RH, Hatala MP. Increased horizontal angle of the mandibular condyle in abnormal temporomandibular joints. A magneric resonance imaging study. Oral Surg Oral Med Oral Pathol Oral Radiol Endod 72: 359, 1991.

14. Kurita H, Ohtsuka A, Kobayashi H, Kurashina K. Relationship between increased horizontal condylar angle and resorption of the posterior region of the lateral pole of the mandibular condyle in temporomandibular joint internal derangement. Dentomaxillofac Radiol 32: 26, 2003.

15. Kurita H, Ohtsuka A, Kobayashi H, Kurashina K. Alternation of the horizontal mandibular condyle size associated with temporomandibular joint internal derangement in adult females. Dentomaxillofac Radiol 31: 373, 2002.

16. Dolwick MF, Heffer LB, et al. Temporomandibular joint surgery. In parameters of care for oral and maxillofacial surgery. A guide for practice, monitoring and evaluation (American Association of Oral and Maxillofacial Surgeons. Parameters of Care-92). J Oral Maxillofac Surg 54:121, 1992.

17. Kurita H, Uehara S, Yokochi M, Nakatsuka A, Kobayashi H, Kurashina K. A long-term follow-up study of radiographically evident degenerative changes in the temporomandibular joint with 
different conditions of disk displacement. Int J Oral Maxillofac Surg 35: 49, 2006.

18. de Leeuw, Boering G, Stegenga B, de Bont LGM. Radiographic signs of temporomandibular joint osteoarthrosis and internal derangement 30 years after nonsurgical treatment. Oral Surg Oral Med Oral Pathol Oral Radiol Endod 79: 382, 1995.

19. Rasmussen OC. Temporomandibular arthropathy: clinical radiologic and therapeutic aspects with emphasis on diagnosis. Int J Oral Surg 12: 365,1983 .

20. Kurita H, Uehara S, Sakai H, Kamata T, Kurashina K. Radiographic follow-up of diseased temporomandibular joint. Oral Surg Oral Med Oral Pathol Oral Radiol Endod 100: 427, 2005.

21. Casares G, Benito C, de la Hoz JL, Benito C. Treatment of TMJ static disk with arthroscopic lysis and lavage: a comparison between MRI arthroscopic findings and clinical results. Cranio 17:49, 1999.

22. Sanroman JF. Closed lock (MRI fixed disc): a comparison of arthrocentesis and arthroscopy. Int J Oral Maxillofac Surg 33:344, 2004.

23. Murakami K, Moriya Y, Goto K, Segami N. Four-year follow-up study of temporomandibular joint arthroscopic surgery for advanced stage internal derangement. J Oral Maxillofac Surg 54:285, 1996. 


\section{Captions for illustrations}

Fig. 1. Methods to describe TMJ disk position (1A), morphology of the articular eminence (1B), horizontal condylar angle (1C), and horizontal size of the condyle (1D)

1A: Measurement of disk position. A tangent is drawn from the lower edge of the articular tubercle (t) to the upper edge of the porus acusticus externus (p). Perpendicular line are drawn from the posterior edge of the disk to the tangent (d). The distances t-p and t-d are measured, and disk position is calculated and recorded as $\mathrm{t}-\mathrm{d} / \mathrm{t}-\mathrm{p}$.

1B: Four types of articular eminence were identified in this study. a: box-type, b: sigmoid-type, c: flattened-type, d: deformed-type.

1C: Measurement of horizontal condylar angle. A transversal line is drawn between the most posterior parts of the left and right condyles. The angle between the transversal line and the line through the lateral and medial poles of the condyle constitutes a horizontal condylar angle (hca).

1D: Measurements of the horizontal size of the condyle. A line is drawn through medial (m) and lateral poles (1) of the condyle. A perpendicular line is drawn to bisect the line $\mathrm{m}-1$, and this intersects the anterior (a) and the posterior edge of the condyle. The distances $m-1$ and a-p are measured in millimeters. 
Table 1. Sex distribution, age, time interval between initial and follow-up examination, and results of initial examination, and outcome of treatments in each group

\begin{tabular}{|c|c|c|}
\hline & $\begin{array}{l}\text { Arthroscopic joints } \\
\text { (11 joints in } 9 \text { patients) }\end{array}$ & $\begin{array}{l}\text { Non-surgical joints } \\
\text { (24 joints in } 19 \text { patients) }\end{array}$ \\
\hline Sex (Male : Female) & $1: 8$ & $5: 14$ \\
\hline Age at initial visit (mean \pm SD) & $40.4 \pm 14.6$ years & $34.3 \pm 15.3$ years \\
\hline $\begin{array}{l}\text { Time interval between } \\
\text { the examinations } \boldsymbol{*}^{\mathbf{1}}\end{array}$ & $47.7 \pm 36.3$ months & $94.5 \pm 23.5$ months \\
\hline \multicolumn{3}{|l|}{ Results of initial examination } \\
\hline Disk displacement & NDD 2, AWR 3, AWOR 6 & NDD 4, AWR 7, AWOR 13 \\
\hline Disk position & $0.31 \pm 0.14$ & $0.32 \pm 0.11$ \\
\hline Morphological change of disk & Normal 6, Mild 3, Severe 2 & Normal 9, Mild 9, Severe 6 \\
\hline Disk mobility $^{* 2}$ & Mobile 2, Fixed 9 & Mobile 22, Fixed 2 \\
\hline OA change of the condyle & Absence 3, Presence 8 & Absence 14, Presence 10 \\
\hline $\begin{array}{l}\text { OA change of the articular } \\
\text { eminence }\end{array}$ & Absence 6, Presence 5 & Absence 19, Presence 5 \\
\hline $\begin{array}{l}\text { Resorption of the lateral part } \\
\text { of the condyle }\end{array}$ & $\begin{array}{l}\text { Absence 3, Presence } 6 \\
\text { (unknown2) }^{* 3}\end{array}$ & $\begin{array}{l}\text { Absence 14, Presence } 6 \\
\text { (unknown 4) }^{* 3}\end{array}$ \\
\hline Horizontal condylar angle & $20.2 \pm 11.8$ degrees & $18.1 \pm 9.8$ degrees \\
\hline \multicolumn{3}{|l|}{ Horizontal condylar size } \\
\hline Medio-lateral dimension & $17.0 \pm 2.4 \mathrm{~mm}$ & $17.6 \pm 4.1 \mathrm{~mm}$ \\
\hline Antero-posterior dimension & $6.8 \pm 1.9 \mathrm{~mm}$ & $7.9 \pm 1.7 \mathrm{~mm}$ \\
\hline Outcome of the treatments ${ }^{* 2,4}$ & Good 6, Fair 3 & Good 19 \\
\hline
\end{tabular}

*1: There was a statistically significant difference between groups (Mann-Whitney’s U-test, $\mathrm{p}<0.05)$.

*2: There was a statistically significant difference between the groups (Fisher's exact probability test, $\mathrm{p}<0.05)$.

*3: The outline of the condyle could not be defined due to a superimposition of the articular eminence, a consequence of limited mouth opening.

*4: According to the criteria reported by American Association of Oral and Maxillofacial Surgeons (1992) 
Table 2. Comparison of interval change in each finding between the joints with arthroscopic surgery (arthroscopic joints) and those with non-surgical treatment (non-surgical joints)

\section{Improve No change Progress}

\section{Disk displacement}

$\begin{array}{llll}\text { Arthroscopic joints } & 1 \text { joint } & 9 \text { joints } & 1 \text { joint } \\ \text { Non-surgical joints } & 4 \text { joints } & 18 \text { joints } & 2 \text { joints }\end{array}$

Morphological change of the TMJ disk

$\begin{array}{llll}\text { Arthroscopic joints } & 1 \text { joint } & 5 \text { joints } & 5 \text { joints } \\ \text { Non-surgical joints } & 3 \text { joints } & 12 \text { joints } & 9 \text { joints }\end{array}$

Disk mobility ${ }^{* 1}$

$\begin{array}{llll}\text { Arthroscopic joints } & 5 \text { joints } & 5 \text { joints } & 1 \text { joint } \\ \text { Non-surgical joints } & 1 \text { joint } & 23 \text { joints } & 0 \text { joint }\end{array}$

OA change of the condyle

$\begin{array}{llll}\text { Arthroscopic joints } & 2 \text { joints } & 8 \text { joints } & 1 \text { joint } \\ \text { Non-surgical joints } & 4 \text { joints } & 15 \text { joints } & 5 \text { joints }\end{array}$

OA change of the articular eminence

$\begin{array}{llll}\text { Arthroscopic joints } & 2 \text { joints } & 8 \text { joints } & 1 \text { joint } \\ \text { Non-surgical joints } & 0 \text { joint } & 21 \text { joints } & 3 \text { joints }\end{array}$

Resorption of the lateral part of the condyle ${ }^{* 2}$

$\begin{array}{llll}\text { Arthroscopic joints } & 0 \text { joint } & 7 \text { joints } & 0 \text { joint } \\ \text { Non-surgical joints } & 3 \text { joints } & 14 \text { joints } & 3 \text { joints }\end{array}$

OA: osteoarthritic/osteoarthrotic

*1: There was a significant difference in distribution between the groups (Goodness test of fit for chi-square, $\mathrm{p}<0.05$ ).

*2: The outline of the condyle could not be defined at either initial or follow-up examination in 4 joints of arthroscopic and in 4 joints of non-surgical joints due to a superimposition of the articular eminence, a consequence of limited mouth opening. 
Table 3. Comparison of mean amount of change in disk position, horizontal condylar angle, and size between the joints with arthroscopic surgery (arthroscopic joints) and those with non-surgical treatment (non-surgical joints)

\begin{tabular}{lll}
\hline & Arthroscopic joints (11) & Non-surgical joints (24) \\
\hline Disk position & -0.04 (SD 0.06) & -0.00 (SD 0.09) \\
Horizontal condylar angle & -1.50 degrees (SD 6.19) & 1.46 (SD 10.18) \\
Horizontal condylar size & & \\
Medio-lateral dimension & $-0.69 \mathrm{~mm}(\mathrm{SD} 2.41)$ & 0.44 (SD 2.88) \\
Antero-posterior dimension & $-0.58 \mathrm{~mm}(\mathrm{SD} 1.17)$ & -0.16 (SD 2.06) \\
\hline
\end{tabular}

SD: standard deviation 
Fig. 1

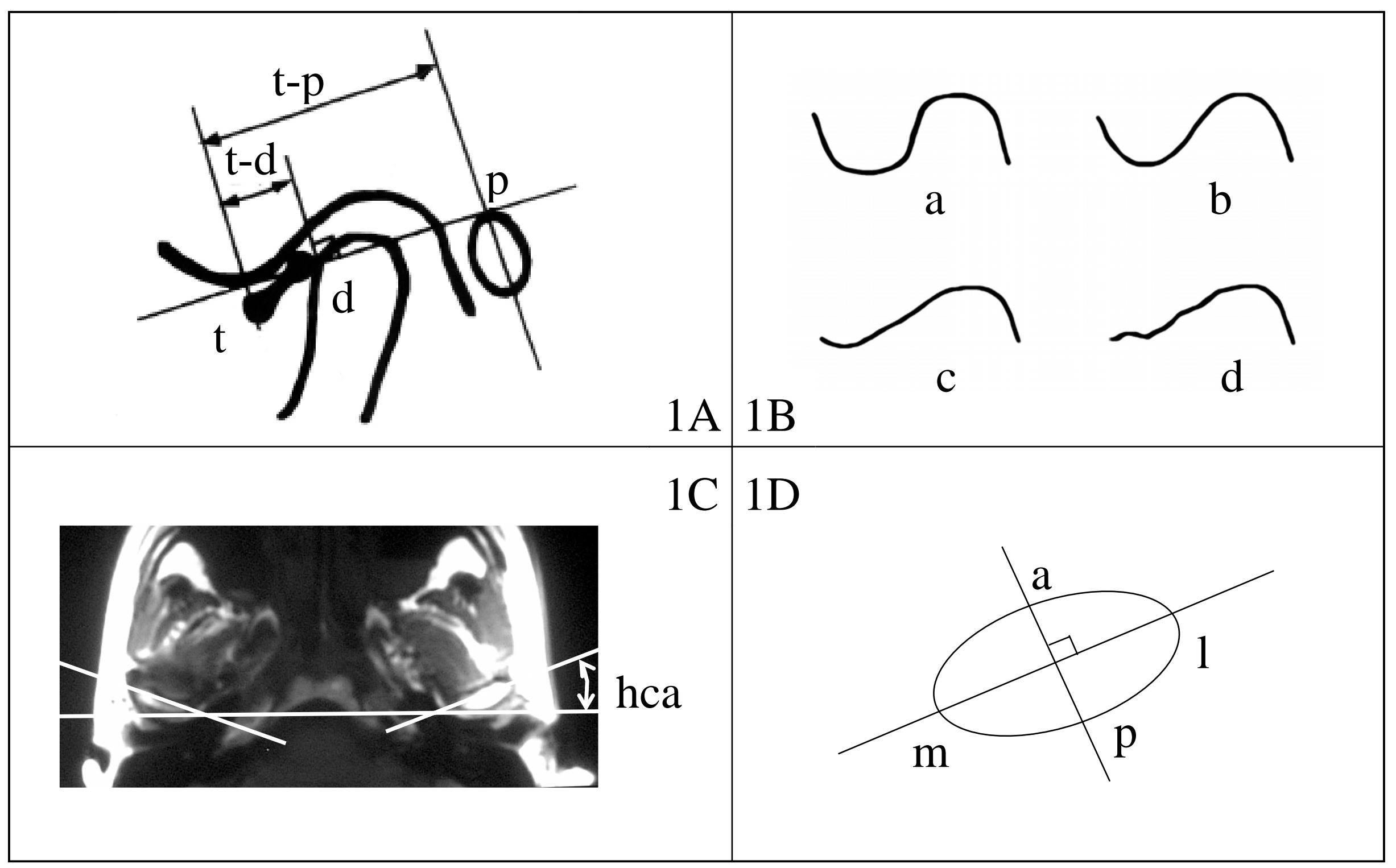

